\title{
EDITORIAL
}

\section{El Ejercicio Físico en la Postmenopausia}

Es bien sabido que en la Postmenopausia, una de las complicaciones más importantes luego de la hipertensión arterial, es la osteoporosis, cuya expresión fisiopatológica es la disminución del contenido mineral óseo, (CMO), lo que predispone a fracturas patológicas en sitios bien establecidos: tercio distal del radio, columna lumbosacra, tercio proximal del fémur. La incapacidad y los costos de atención que ello implica, sugiere la necesidad de implementar acciones desde el punto de vista preventivo, entre las cuales se incluye la actividad física.

El ejercicio está inscrito como una medida de prevención no solamente para la osteoporosis postmenopáusica (OPPM), sino para muchas otras situaciones de la salud; sin embargo, sus características y bases científicas no siempre son claras. En este pequeño escrito, se aspira a fundamentar la acción que tiene la fibra muscular sobre el tejido óseo, para finalmente recalcar la importancia del ejercicio en el manejo de la mujer peri y postmenopáusica.

El hueso, al igual que otros tejidos, consta de células, fibras y sustancia fundamental, pero a diferencia de los demás, sus componentes extracelulares están calcificados, haciendo de él una estructura dura y resistente, excelentemente dotada para sus funciones de sostén y protección.

Las características químicas de la matriz orgánica y mineral, le confieren al hueso la resistencia a la tracción y a la compresión, al tiempo que gracias a sus propiedades físicas, le permiten que goce de elasticidad; en este proceso, el colágeno proporciona al hueso la capacidad para resistir la tensión. La tensión ósea debe ser desmembrada en sus componentes de dirección, magnitud y tipo (tracción, compresión, cizallamiento e incurvación). La disposición trabecular del hueso esponjoso, es favorecida por las fuerzas de peso y en menor grado por las de tensión. Un aumento del stréss mecánico, origina hipertrofia de las trabéculas existentes. Este es uno de los principios por medio de los cuales el ejercicio físico proporciona mejor calidad de hueso en la OPPM.

El tejido óseo es sensible a los requerimientos que se le hacen y reacciona ante ellos de tal modo, que todo cambio en la función del hueso, es seguido en mayor o menor medida por un cambio preciso en la arquitectura interna. Wolff demostró que el tejido óseo se reorganiza cuando se aplican cambios de fuerzas mecánicas, según leyes matemáticas precisas, que incluyen el principio por medio del cual los huesos podrían aumentar o disminuir su masa de acuerdo al tipo y magnitud de las fuerzas a que están sometidos; la dirección en que actúan tales fuerzas, hace referencia a las propiedades mecánicas del hueso.

El afecto más importante de estas fuerzas en la estructura ósea, es la deformación mecánica, en donde el hueso actúa a manera de transductor, convirtiendo la energía mecánica en eléctrica y provocando como consecuencia un cambio de potencial y la creación de un flujo eléctrico cuyo signo depende de la dirección de la fuerza mecánica aplicada. El colágeno o la interfase colágeno-cristal de apatita, parece ser el responsable de esas cualidades piezoeléctricas y el efecto de cambio de potencial es una despolarización de la membrana de las células, que modifica la actividad metabólica celular, favoreciendo el proceso de remodelado óseo.

La actividad física cumple papel preponderante en el mecanismo de mantenimiento de la estructura y en la dinámica del sistema esquelético, al brindar las fuerzas necesarias para producir deformaciones que mantienen en equilibrio el remodelado óseo. Diversos estudios han demostrado la relación positiva existente entre la actividad física (fuerza muscular y capacidad aeróbica) y la masa ósea. Dos factores parecen ser los primariamente responsables del efecto del ejercicio en el remodelado óseo:

- La Carga de Peso, al proveer una fuerza mecánica en el hueso, que estimula la función osteoblástica; el stréss mecánico producido sobre el hueso por el ejercicio de carga de peso, resulta en su hipertrofia.

- La Contracción Muscular: utilizando los mismos principios de los efectos mecánicos de las fuerzas estimulando la función osteoblástica, las fuerzas ejercidas por una contracción muscular, producen la misma respuesta metabólica en el hueso.

De esta manera y teniendo en cuenta los beneficios potenciales que cualquier programa de ejercicio físico pueda tener, también se deberán tener en cuenta los efectos secundarios que podrían producirse, como resultado de un inadecuado entrenamiento o de la realización de actividades para las cuales no se esté lo suficientemente preparado el sujeto partícipe; sin embargo y dado que el ejercicio como tal no ejerce efectos secundarios, sino todo lo contrario, que provee elementos que favorecen la actitud de quien participa en él, se deberá asegurar niveles adecuados de intensidad, frecuencia y duración de los mismos, que dependerán del grupo poblacional particular.

\author{
Edgar Cortés Reyes \\ Profesor. Universidad Nacional de Colombia.
}

\section{BIBLIOGRAFIA}

- Aloia J. Exercise and the regulation of bone mass. Arch. Intern. Med. 1989; (10):2170-2171

- Stillman R. y col. Physical activity and bone in women aged 30 to 85 years. Med. and sci. Sports and Exercise, 18 1986; (5):576-580

- Aisenbrey J. Exercise in the prevention and management of osteoporosis. Phys. Ther., 67 1987; (7):1100-1104

- Basset C.A. Generation of electrical potentials by bone in response to mechanical stress. Science 137. 1962; 1063-1064

- Krolner B. Physical exercise as prophylaxis against involutional vertebral bone loss; controlled trial. Clin Sci. 1983; 64:541-546 